\title{
Resonant Photon Tunneling Enhancement of the van der Waals Friction
}

\author{
A. I. Volokitin ${ }^{1,2}$ and B. N. J. Persson ${ }^{1}$ \\ ${ }^{1}$ Institut für Festkorperforschung, Forschungszentrum, Jülich D-52425, Germany \\ ${ }^{2}$ Samara State Technical University, 443100 Samara, Russia \\ (Received 1 May 2003; published 4 September 2003)
}

\begin{abstract}
We study the van der Waals friction between two flat metal surfaces in relative motion. For good conductors, we find that normal relative motion gives a much larger friction than for parallel relative motion. The friction may increase by many orders of magnitude when the surfaces are covered by adsorbates, or can support low-frequency surface plasmons. In this case, the friction is determined by resonant photon tunneling between adsorbate vibrational modes, or surface plasmon modes.
\end{abstract}

DOI: 10.1103/PhysRevLett.91.106101

PACS numbers: 68.35.Af, 68.37.Ps, 73.20.Mf

A great deal of attention has been devoted to noncontact friction between nanostructures, including, for example, the frictional drag force between two-dimensional quantum wells [1-4], and the friction force between an atomic force microscope tip and a substrate [5-9].

In noncontact friction, the bodies are separated by a potential barrier thick enough to prevent electrons or other particles with a finite rest mass from tunneling across it, but allowing interaction via the long-range electromagnetic field, which is always present in the gap between bodies. The presence of inhomogeneous tipsample electric fields is difficult to avoid, even under the best experimental conditions [7]. For example, even if both the tip and the sample were metallic single crystals, the tip would still have corners present and more than one crystallographic plane exposed. The presence of atomic steps, adsorbates, and other defects will also contribute to the inhomogeneous electric field. The electric field can be easily changed by applying a voltage between the tip and the sample.

The electromagnetic field can also be created by the fluctuating current density, due to thermal and quantum fluctuations inside the solids. This fluctuating electromagnetic field is always present close to the surface of any body, and consists partly of traveling waves and partly of evanescent waves which decay exponentially with the distance away from the surface of the body. The fluctuating electromagnetic field originating from the fluctuating current density inside the bodies gives rise to the wellknown long-range attractive van der Waals interaction between two bodies [10]. If the bodies are in relative motion, the same fluctuating electromagnetic field will give rise to a friction which is frequently named as the van der Waals friction.

Although the dissipation of energy connected with the noncontact friction always is of electromagnetic origin, the detailed mechanism is not totally clear, since there are several different mechanisms of energy dissipation connected with the electromagnetic interaction between bodies. First, the electromagnetic field from one body will penetrate into the other body, and induce an electric current. In this case, friction is due to Ohmic losses inside the bodies. Another contribution to friction from the electromagnetic field is associated with the time-dependent stress acting on the surface of the bodies. This stress can excite acoustic waves, or induce time-dependent deformations which may result in a temperature gradient. It can also induce motion of defects either in the bulk or on the surface of the bodies. The contribution to friction due to nonadiabatic heat flow, or motion of defects, is usually denoted as internal friction.

It is very worthwhile to get a better understanding of different mechanisms of noncontact friction because of its practical importance for ultrasensitive force detection experiments. This is because the ability to detect small forces is inextricably linked to friction via the fluctuationdissipation theorem. For example, the detection of single spins by magnetic resonance force microscopy, which has been proposed for three-dimensional atomic imaging [11] and quantum computation [12], will require force fluctuations to be reduced to unprecedented levels. In addition, the search for quantum gravitation effects at a short length scale [13] and future measurements of the dynamical Casimir forces [14] may eventually be limited by noncontact friction effects.

Recently, Gotsmann and Fuchs [6] observed longrange noncontact friction between an aluminum tip and a gold (111) surface. The friction force $F$ acting on the tip is proportional to the velocity $v, F=\Gamma v$. For motion of the tip normal to the surface, the friction coefficient $\Gamma(d)=b d^{-3}$, where $d$ is the tip-sample spacing and $b=$ $\left(8.0_{-4.5}^{+5.5}\right) \times 10^{-35} \mathrm{Ns} \mathrm{m}^{2}$ [6]. Later, Stipe et al. [7] observed a noncontact friction effect between a gold surface and a gold-coated cantilever as a function of tip-sample spacing $d$, temperature $T$, and bias voltage $V$. For vibration of the tip parallel to the surface, they found $\Gamma(d)=$ $\alpha(T)\left(V^{2}+V_{0}^{2}\right) / d^{n}$, where $n=1.3 \pm 0.2$ and $V_{0} \sim 0.2 \mathrm{~V}$. At $295 \mathrm{~K}$, for the spacing $d=100 \AA$, they found $\Gamma=$ $1.5 \times 10^{-13} \mathrm{~kg} \mathrm{~s}^{-1}$ which is $\sim 500$ times smaller than 
reported in Ref. [6] at the same distance using a parallel cantilever configuration.

In a recent Letter, Dorofeev et al. [5] claim that the noncontact friction effect observed in [5,6] is due to Ohmic losses mediated by the fluctuating electromagnetic field . This result is controversial, however, since the van der Waals friction has been shown [15-18] to be many orders of magnitude smaller than the friction observed by Dorofeev et al. Presently, the origin of the difference in magnitude and distance dependence of the long-range noncontact friction effect observed in [6,7] is not well understood.

In order to improve the basic understanding of noncontact friction, in this Letter we present new results for van der Waals friction. In [16] we developed a theory of van der Waals friction for surfaces in parallel relative motion. Here we generalize the theory to include also the case when the surfaces are in normal relative motion, and we show that there is a drastic difference between these two cases. Thus, for normal relative motion of clean good conductor surfaces, the friction is many orders of magnitude larger than for parallel relative motion, but still smaller than observed experimentally. Another enhancement mechanism of the noncontact friction can be connected with resonant photon tunneling between states localized on the different surfaces. Recently, it was dis- covered that resonant photon tunneling between surface plasmon modes gives rise to extraordinary enhancement of the optical transmission through subwavelength hole arrays [19]. The same surface modes enhancement can be expected for van der Waals friction if the frequency of these modes is sufficiently low to be excited by thermal radiation. At room temperature, only the modes with frequencies below $\sim 10^{13} \mathrm{~s}^{-1}$ can be excited. For normal metals, surface plasmons have much too high frequencies; at thermal frequencies, the dielectric function of normal metals becomes nearly purely imaginary, which exclude surface plasmon enhancement of the van der Waals friction for good conductors. However, surface plasmons for semiconductors are characterized by much smaller frequencies and damping constants, and they can give an important contribution to van der Waals friction. Other surface modes which can be excited by thermal radiation are adsorbate vibrational modes. Especially for parallel vibrations, these modes may have very low frequencies.

Recently [15], we developed a theory of the van der Waals friction between two semi-infinite bodies, moving parallel to each other. We have generalized this theory to two semi-infinite bodies, moving normal to each other. The frictional stress, $\sigma$, is proportional to the velocity $v$, $\sigma=\gamma v$. For the separation $d<c h / k_{B} T$, we get the coefficient of friction $\gamma_{\perp}$ [20]:

$$
\begin{aligned}
\gamma_{\perp}=\frac{\hbar}{\pi^{2}} \int_{0}^{\infty} d \omega \int_{0}^{\infty} d q q^{3}\left(-\frac{\partial n(\omega)}{\partial \omega}\right) e^{-2 q d} \times\{ & \left(\left(\operatorname{Im} R_{1 p}+e^{-2 q d}\left|R_{1 p}\right|^{2} \operatorname{Im} R_{2 p}\right)\left(\operatorname{Im} R_{2 p}+e^{-2 q d}\left|R_{2 p}\right|^{2} \operatorname{Im} R_{1 p}\right)\right. \\
& \left.\left.+e^{-2 q d}\left[\operatorname{Im}\left(R_{1 p} R_{2 p}\right)\right]^{2}\right) \frac{1}{\left|1-e^{-2 q d} R_{1 p} R_{2 p}\right|^{4}}+[p \rightarrow s]\right\},
\end{aligned}
$$

where the Bose-Einstein factor

$$
n(\omega)=\frac{1}{e^{\hbar \omega / k_{B} T}-1} .
$$

The symbol $[p \rightarrow s]$ in (1) denotes the term which is obtained from the first one by replacement of the reflection amplitude $R_{p}(\omega)$ for $p$-polarized waves by the reflection amplitude $R_{s}(\omega)$ for $s$-polarized waves.

The friction coefficient for two flat surfaces in parallel relative was obtained by us previously [15], and the contribution to the friction from the evanescent electromagnetic waves is given by

$$
\begin{aligned}
\gamma_{\|}= & \frac{\hbar}{2 \pi^{2}} \int_{0}^{\infty} d \omega \int_{0}^{\infty} d q q^{3}\left(-\frac{\partial n(\omega)}{\partial \omega}\right) e^{-2 q d} \\
& \times \frac{\operatorname{Im} R_{1 p} \operatorname{Im} R_{2 p}}{\left|1-e^{-2 q d} R_{1 p} R_{2 p}\right|^{2}}+[p \rightarrow s] .
\end{aligned}
$$

There is a principal difference between the friction coefficient for normal and parallel relative motion, related to the denominators of the integrand in (1) and (3). These detominators are due to multiple scattering of evanescent electromagnetic waves from opposite surfaces. The resonant condition corresponds to the case when these detominators are small. For two identical surfaces and $R_{i} \ll 1 \leq R_{r}$, where $R_{i}$ and $R_{r}$ are the imaginary and real parts, respectively, this corresponds to the resonant condition $R_{r}^{2} \exp (-2 q d) \approx 1$. At resonance, the integrand in (1) has a large additional factor $\sim 1 / R_{i}^{2}$ in comparison to the case of parallel relative motion. The resonance condition can be fulfilled even for the case when $\exp (-2 q d) \ll 1$ because for evanescent electromagnetic waves there is no restriction on the magnitude of real part or the modulus of $R$. This opens up the possibility of resonant denominators for $R_{r}^{2} \gg 1$.

The reflection amplitude $R_{p}$, which takes into account the contribution from an adsorbate layer, is given by [21]

$$
R_{p}=\frac{q-s / \epsilon+4 \pi n_{a} q\left[s \alpha_{\|} / \epsilon+q \alpha_{\perp}\right]}{q+s / \epsilon+4 \pi n_{a} q\left[s \alpha_{\|} / \epsilon-q \alpha_{\perp}\right]},
$$

where

$$
s=\sqrt{q^{2}-\left(\frac{\omega}{c}\right)^{2} \epsilon}
$$

and where $\alpha_{\|}$and $\alpha_{\perp}$ are the polarizabilities of adsorbates in a direction parallel and normal to the surface, 
respectively. $\epsilon$ is the bulk dielectric function and $n_{a}$ is the concentration of adsorbates. For clean surfaces $n_{a}=0$, and in this case formula (4) reduces to the well-known Fresnel formula.

Let us first consider two identical metals described by the dielectric function:

$$
\epsilon=1-\frac{\omega_{p}^{2}}{\omega\left(\omega+i \tau^{-1}\right)},
$$

where $\tau$ is the relaxation time and $\omega_{p}$ the plasma frequency. For good conductors at thermal frequencies $R_{p i} \ll 1$ and $R_{p r} \approx 1$. Thus, an enhancement in friction is possible only for very small $q \ll 1 / d$. Analysis shows that integral (1) has a $1 / q^{3}$ singularity and the main contribution to the integral (1) comes from the vicinity of this singularity. For two bodies, moving parallel to each other, the integral has only a logarithmic singularity for small $q$, and the main contribution comes from the nonresonant region with $q \sim 1 / d$. Thus, for clean surfaces of good conductors, for normal relative motion the van der Waals friction will be on many order of magnitude larger than for parallel relative motion. Figure 1 illustrates this situation for two copper surfaces and $T=$ $273 \mathrm{~K}$. However, the van der Waals friction in this case is too small in comparison with experimental data. Thus, for $d=1 \mathrm{~nm}$, the friction $\gamma_{\perp \text { theor }} \sim 10^{-4} \mathrm{~kg} \mathrm{~s}^{-1} \mathrm{~m}^{-2}$. For an atomic force microscope tip, one can estimate $\Gamma \approx \gamma S$, where $S \approx R d$ is an effective surface area of the tip with a radius of curvature $R$. For the tip with $R \sim 1 \mu \mathrm{m}$, the friction coefficient $\Gamma_{\text {theor }} \sim 10^{-19} \mathrm{~kg} \mathrm{~s}^{-1}$, while from the experimental data at the same distance one can deduce the friction coefficient $\Gamma_{\exp } \sim 10^{-12} \mathrm{~kg} \mathrm{~s}^{-1}$ [7].

Resonant photon tunneling enhancement of the van der Waals friction is possible for two semiconductor surfaces which can support low-frequency surface plasmon

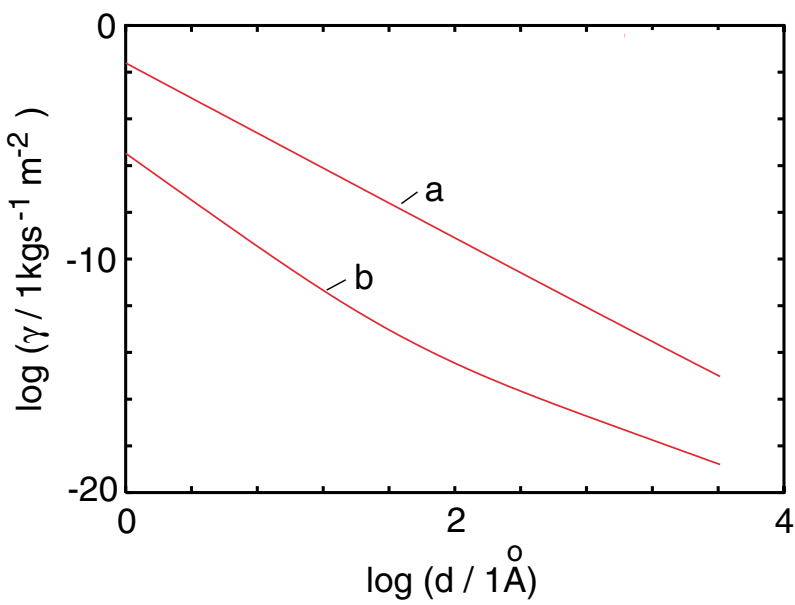

FIG. 1 (color online). The friction coefficient for two (clean) surfaces in $(a)$ normal and $(b)$ parallel relative motion, as a function of separation $d . T=273 \mathrm{~K}$ and with parameters chosen to correspond to copper $\left(\tau^{-1}=2.5 \times 10^{13} \mathrm{~s}^{-1}, \omega_{p}=\right.$ $1.6 \times 10^{16} \mathrm{~s}^{-1}$ ). (The $\log$ function is with basis 10.) modes. As an example, we consider two clean surfaces of silicon carbide ( $\mathrm{SiC})$. The optical properties of this material can be described using an oscillator model [22]:

$$
\epsilon(\omega)=\epsilon_{\infty}\left(1+\frac{\omega_{L}^{2}-\omega_{T}^{2}}{\omega_{T}^{2}-\omega^{2}-i \Gamma \omega}\right),
$$

with $\epsilon_{\infty}=6.7, \quad \omega_{L}=1.8 \times 10^{14} \mathrm{~s}^{-1}, \quad \omega_{T}=1.49 \times$ $10^{14} \mathrm{~s}^{-1}$, and $\Gamma=8.9 \times 10^{11} \mathrm{~s}^{-1}$. The frequency of surface plasmons is determined by condition $\epsilon_{r}\left(\omega_{p}\right)=-1$ and from (7) we get $\omega_{p}=1.78 \times 10^{14} \mathrm{~s}^{-1}$. In Fig. 2, we plot the friction coefficient $\gamma(d)$ : Note that the friction between the two semiconductor surfaces is several order of magnitude larger than between two clean good conductor surfaces.

Another enhancement mechanism is connected with resonant photon tunneling between adsorbate vibrational modes localized on different surfaces. In this case, the real part of the reflection amplitude $R_{p}$ can be much larger than unity, and the resonant condition $\exp (-2 q d) R_{p}^{2} \sim 1$ can be fulfilled even for large $q$, giving rise to large enhancement of friction. As an example, let us consider ions with charge $e^{*}$ adsorbed on metal surfaces. The polarizability for ion vibration normal to the surface is given by

$$
\alpha_{\perp}=\frac{e^{* 2}}{M\left(\omega_{\perp}^{2}-\omega^{2}-i \omega \eta_{\perp}\right)},
$$

where $\omega_{\perp}$ is the frequency of the normal adsorbate vibration, and $\eta_{\perp}$ is the damping constant. In Eq. (4), the contribution from parallel vibrations is reduced by the small factor $1 / \epsilon$. However, the contribution of parallel vibrations to the van der Waals friction can nevertheless be important due to the indirect interaction of parallel

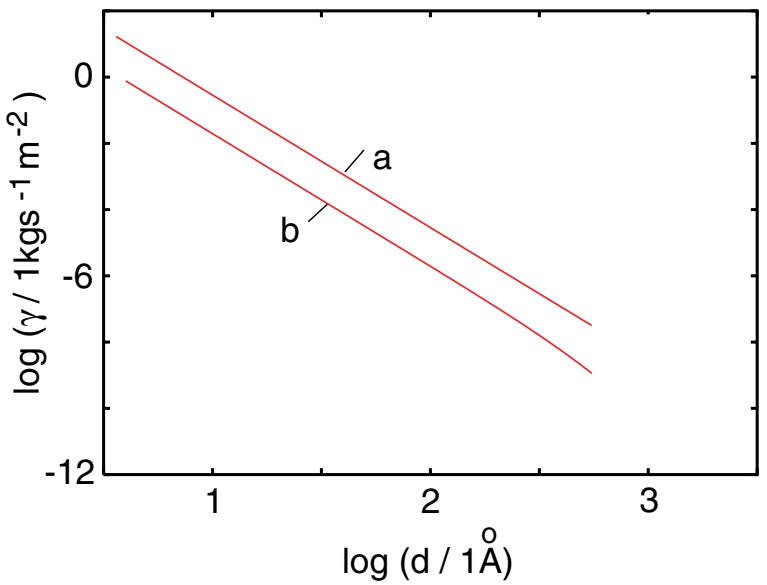

FIG. 2 (color online). The friction coefficient for two clean semiconductor surfaces in $(a)$ normal and $(b)$ parallel relative motion, as a function of the separation $d . T=300 \mathrm{~K}$ and with parameters chosen to correspond to a surfaces of silicon carbide (SiC) (see text for explanation). (The log function is with basis 10 .) 


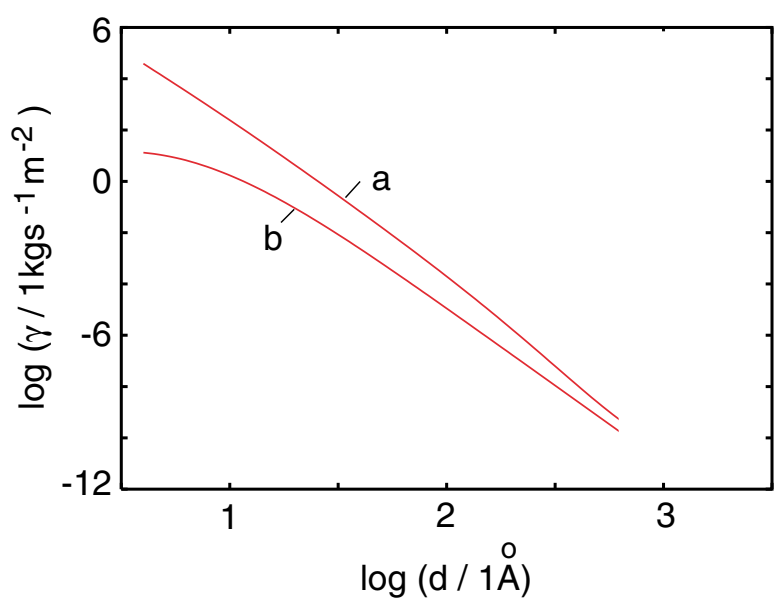

FIG. 3 (color online). The friction coefficient for two surface covered by adsorbates in $(a)$ normal and $(b)$ parallel relative motion, as a function of the separation $d . T=273 \mathrm{~K}$ and with parameters chosen to correspond to $\mathrm{K} / \mathrm{Cu}(001)$ [24] $\left(\omega_{\perp}=\right.$ $1.9 \times 10^{13} \mathrm{~s}^{-1}, \quad \omega_{\|}=4.5 \times 10^{12} \mathrm{~s}^{-1}, \quad \eta_{\|}=2.8 \times 10^{10} \mathrm{~s}^{-1}$, $\eta_{\perp}=1.6 \times 10^{12} \mathrm{~s}^{-1}, e^{*}=0.88 e$ ). (The $\log$ function is with basis 10.$)$

adsorbate vibration with the electric field, via the metal conduction electron [23]. Thus, the small parallel component of the electric field will induce a strong electric current in the metal. The drag force between the electron flow and adsorbates can induce adsorbate vibrations parallel to the surface. This gives the polarizability:

$$
\alpha_{\|}=\frac{\epsilon-1}{n} \frac{e^{*}}{e} \frac{\omega \eta_{\|}}{\left(\omega_{\|}^{2}-\omega^{2}-i \omega \eta_{\|}\right)},
$$

where $n$ is the conduction electron concentration. As an illustration, in Fig. 3 we show the coefficient of friction for the two $\mathrm{Cu}(001)$ surfaces covered by a low concentration of potassium atoms $\left(n_{a}=10^{18} \mathrm{~m}^{-2}\right)$. In the $q$ integral in Eq. (1), we used the cutoff $q_{c} \sim \pi / a$ (where $a \approx 1 \mathrm{~nm}$ is the interadsorbate distance) because our microscopic approach is applicable only when the wavelength of the electromagnetic field is larger than double average distance between the adsorbates. In comparison, the friction between two clean surface at separation $d=1 \mathrm{~nm}$ is 7 orders of magnitude smaller. At $d=1 \mathrm{~nm}$, the friction coefficient $\Gamma$ for an atomic force microscope tip with $R \sim 1 \mu \mathrm{m}$ is $\sim 10^{-12} \mathrm{~kg} \mathrm{~s}^{-1}$ ( $\gamma \sim$ $10^{3} \mathrm{~kg} \mathrm{~s}^{-1} \mathrm{~m}^{-2}$, see Fig. 3); this is of the same order of magnitude as the observed friction [7].

In this Letter, we have shown that the van der Waals friction can be enhanced by several orders of magnitude when the material involved supports low-frequency adsorbate vibrational modes or surface plasmon modes. For clean surfaces of good conductors, friction is several order of magnitude larger for normal relative motion as compared to parallel relative motion. These results should have a broad application in noncontact friction microscopy, and in the design of new tools for studying adsorbate vibrational dynamics.

A. I.V. acknowledges financial support from DFG. B. N. J.P. acknowledges support from the European Union Smart Quasicrystals project.

[1] T. J. Gramila, J. P. Eisenstein, A. H. Macdonald, L. N. Pfeiffer, and K.W. West, Phys. Rev. Lett. 66, 1216 (1991);Surf. Sci. 263, 446 (1992).

[2] T. J. Gramila, J. P. Eisenstein, A. H. Macdonald, L. N. Pfeiffer, and K.W. West, Phys. Rev. B 47, 12957 (1993); Physica (Amsterdam) 197B, 442 (1994).

[3] U. Sivan, P. M. Solomon, and H. Shrikman, Phys. Rev. Lett. 68, 1196 (1992).

[4] A. I. Volokitin and B. N. J. Persson, J. Phys. Condens. Matter 13, 859 (2001).

[5] I. Dorofeev, H. Fuchs, G. Wenning, and B. Gotsmann, Phys. Rev. Lett. 83, 2402 (1999).

[6] B. Gotsmann and H. Fuchs, Phys. Rev. Lett. 86, 2597 (2001).

[7] B. C. Stipe, H. J. Mamin, T. D. Stowe, T.W. Kenny, and D. Rugar, Phys. Rev. Lett. 87, 096801 (2001).

[8] H. J. Mamin and D. Rugar, Appl. Phys. Lett. 79, 3358 (2001).

[9] P. M. Hoffmann, S. Jeffery, J. B. Pethica, H. Özgür Özer, and A. Oral, Phys. Rev. Lett. 87, 265502 (2001).

[10] I. E. Dzyaloshinskii, E. M. Lifshitz, and L. P. Pitaevskii, Adv. Phys. 10, 165 (1961).

[11] J. A. Sidles et al., Rev. Mod. Phys. 67, 249 (1995).

[12] G. P. Berman et al., Phys. Rev. B 61, 14694 (2000).

[13] N. Arkani-Hamed, S. Dimopoulos, and G. Dvali, Phys. Lett. B 429, 263 (1998); Sci. Am. 283, 62 (2000).

[14] U. Mohideen and A. Roy, Phys. Rev. Lett. 81, 4549 (1998).

[15] A. I. Volokitin and B. N. J. Persson, J. Phys. Condens. Matter 11, 345 (1999); Phys. Low-Dim. Struct. 7/8, 17 (1998).

[16] B. N. J. Persson and A. I. Volokitin, Phys. Rev. Lett. 84, 3504 (2000).

[17] A. I. Volokitin and B. N. J. Persson, Phys. Rev. B 63, 205404 (2001); Phys. Low-Dim. Struct. 5/6, 151 (2001).

[18] A. I. Volokitin and B. N. J. Persson, Phys. Rev. B 65, 115419 (2002).

[19] A. Krishnan et al., Opt. Commun. 200, 1 (2001).

[20] A. I. Volokitin and B. N. J. Persson (to be published).

[21] D. C. Langreth, Phys. Rev. B 39, 10020 (1989).

[22] E. D. Palik, Handbook of Optical Constants of Solids (Academic, San Diego, CA, 1985).

[23] B. N. J. Persson and A. I. Volokitin, Surf. Sci. 310, 314 (1994).

[24] P. Senet et al., Chem. Phys. Lett. 299, 389 (1999). 